\title{
Investigation of the forced Bonhoeffer van der Pol equations via continuation and other methods
}

\author{
R. M. Pennifold ${ }^{1}$ \\ H. S. Sidhu ${ }^{2}$ \\ G. N. Mercer $^{3}$
}

(Received 31 July 2007; revised 07 April 2008)

\begin{abstract}
Previous work used bifurcation diagrams and Lyapunov exponents to explore the dynamics of the forced Bonhoeffer van der Pol equation. Here we use continuation methods to clarify the bifurcations of the periodic orbits which had previously been ambiguous. We discuss the use of numerical methods including Galerkin and the recent 0-1 test for chaos.
\end{abstract}

\section{Contents}

See http://anziamj.austms.org.au/ojs/index.php/ANZIAMJ/article/view/350 for this article, (c) Austral. Mathematical Soc. 2008. Published June 11, 2008. ISSN 1446-8735 
2 Earlier study

C630

2.1 Bifurcation analysis . . . . . . . . . . . . C630

3 Characterising chaotic behaviour via the $0-1$ test

C633

4 Conclusion

C638

References

C638

\section{Introduction}

Here we explore the dynamics of the forced Bonhoeffer van der Pol (BVP) equations

$$
\begin{aligned}
& \frac{d x}{d t}=c\left(x+y-x^{3} / 3-A \cos \omega t\right), \\
& \frac{d y}{d t}=\frac{-1}{c}(x+b y-a) .
\end{aligned}
$$

The control parameters of the system are $a, b, c>0, \omega$ and $A$. Typically $a, b, c$ and $\omega$ are held constant and $A$ is varied between 0 and 1 . These equations are a simplified version of the four variable Hodgkin Huxley (HH) equations [6]. The HH equations are a description of how nerve impulses travel down the axon of a squid nerve cell. Through careful experiments it was determined that one can model the nerve impulses via ion concentrations, the opening and closing of ion channels in the cell membrane and the membrane capacitance [11]. Hodgkin and Huxley [11] discovered that the sodium and potassium ion currents (sodium and potassium being the primary components of membrane currents) are regulated separately by individual ion channels.

A simplified two variable version of the HH equations is known as the Fitzhugh-Nagumo (FN) equations [6]. These equations are closely related to 
the BVP equations (1)-(2). Fitzhugh [6] referred to the FN equations as the Bonhoeffer van der Pol model. Fitzhugh [6] constructed the following model

$$
\begin{aligned}
& \frac{d x}{d t}=c\left(x+y-x^{3} / 3-z\right), \\
& \frac{d y}{d t}=-\frac{1}{c}(x+b y-a),
\end{aligned}
$$

where

$$
1-2 b / 3<a<1, \quad 0<b<1 \text { and } b<c^{2} .
$$

Fitzhugh [6] described $z$ as the stimulus intensity corresponding to the membrane current $I$ from the HH equations. Although Fitzhugh's article describes $z$ as an arbitrary function of time, it was only ever used as a constant (albeit a constant held at different values for different experiments). In the forced BVP system, equations (1)-(2), a sinusoidal forcing term $A \cos \omega t$, is used as the stimulus intensity. We think of this as the nerve membrane subjected to a periodic external stimulus [1]. Probing biological systems with an application of sinusoidal stimulation and comparing experimental data with that of physiological models is one way to better understand the underlying physiological processes [10].

Although equations (1)-(2) are a reduced model, an approximate mapping into the HH model is possible. The fast $x$ variable represents the membrane potential and the slow $y$ variable represents the recovery variable or the potassium conductance [3].

Previous studies of the dynamical behaviour of the forced system (1)-(2), such as Barnes and Grimshaw [1] and references contained therein, primarily used bifurcation diagrams and Lyapunov exponents. However, Croisier and Dauby [4] used continuation of periodic solutions of a system similar to (1)-(2) but with different forcing. It is remarkable how much of the system's dynamics is discernible through application of these tools, combined with a good understanding of nonlinear dynamical systems [1]. As powerful as these techniques are, in some circumstances they are unable to resolve all 
the dynamics of the system. We employ continuation of periodic orbits to resolve these issues. There are several algorithms commonly used to determine periodic solutions of nonlinear dynamical systems, including Galerkin [17], shooting [13] and collocation [5] methods. Often these are used in conjunction with continuation methods to map out a path of periodic solutions as a parameter is varied.

Here we use a Fourier-Galerkin method where we represent the solution of a system of differential equations (DEs) $\dot{x}=f(x, t)$, (such that $f(x, t)=$ $f(x, t+2 \pi)$ ), as a truncated Fourier expansion, the coefficients of which are to be determined. We rewrite the right hand side of the DEs as a Fourier expansion and on discretising the integrals in the Fourier expansion we arrive at a set of algebraic equation in terms of the unknown coefficients, which are solved via Newton-Raphson method [17].

The methods discussed above are essentially numerical; however, there are also analytical methods, such as perturbation, averaging and harmonic balance. Although not treated in this article, for completeness we shall briefly discuss these analytic methods. The perturbation approach works well when the system is weakly nonlinear. In this case begin with a 'nearby' linear solution and successively improve the approximation with any of a number of perturbation techniques. An alternative is to apply a singular perturbation approach similar to Guckenheimer et al. [9], where the dynamics are approximated on the fast and slow manifolds. These methods applied to the BVP system will be investigated in future work. The harmonic balance method has the virtue that it does not require weak nonlinearity, it merely assumes that the solution can be well represented by a harmonic sequence [12].

The system (1)-(2) is known to exhibit chaotic motion. Recently Gottwald and Melbourne [7] developed a technique for detecting chaotic dynamics. This technique applies as easily to experimental data as it does to data generated from integrating known equations. This is a novel approach which exploits ergodic properties of the chaotic attractor [14]. We will apply this technique to the forced BVP system (1)-(2) and compare these results with 
those obtained via Lyapunov exponents, verifying the utility of the method.

\section{Earlier study}

As outlined in the work of Barnes and Grimshaw [1] the bifurcation diagram is a useful way to obtain an overview of a system's dynamics, especially of forced systems. Barnes and Grimshaw [1] demonstrate that the system (1)(2) is capable of a rich array of dynamics including periodic, quasi-periodic, mode locking phenomena and chaotic behaviour, for $0<A<1$.

However, the analysis of Barnes and Grimshaw [1] was limited without performing the continuation of periodic solutions. A case in point is the overlapping solutions with period $4 \pi$ and $6 \pi$ solutions for $0.39<A<0.41$ as seen in Figure 1. Barnes and Grimshaw [1] described this as a blue sky catastrophe where the system instantaneously jumps from one periodic attractor to another as the parameter $A$ is varied. By merely referring to the bifurcation diagrams it is a difficult task to fully understand what is happening at these locations, hence the need for further investigation via periodic continuation and bifurcation analysis using Floquet theory.

Following the work of Ben-Tal et al. [2] we used arc length continuation and test the stability of solutions using Floquet theory [15].

\subsection{Bifurcation analysis}

For brevity we only illustrate the use of continuation methods to the period $4 \pi$ case in detail. However, we used similar tools to uncover the full behaviour of other periodic solution branches which were unclear from the analysis of Barnes and Grimshaw [1]. 


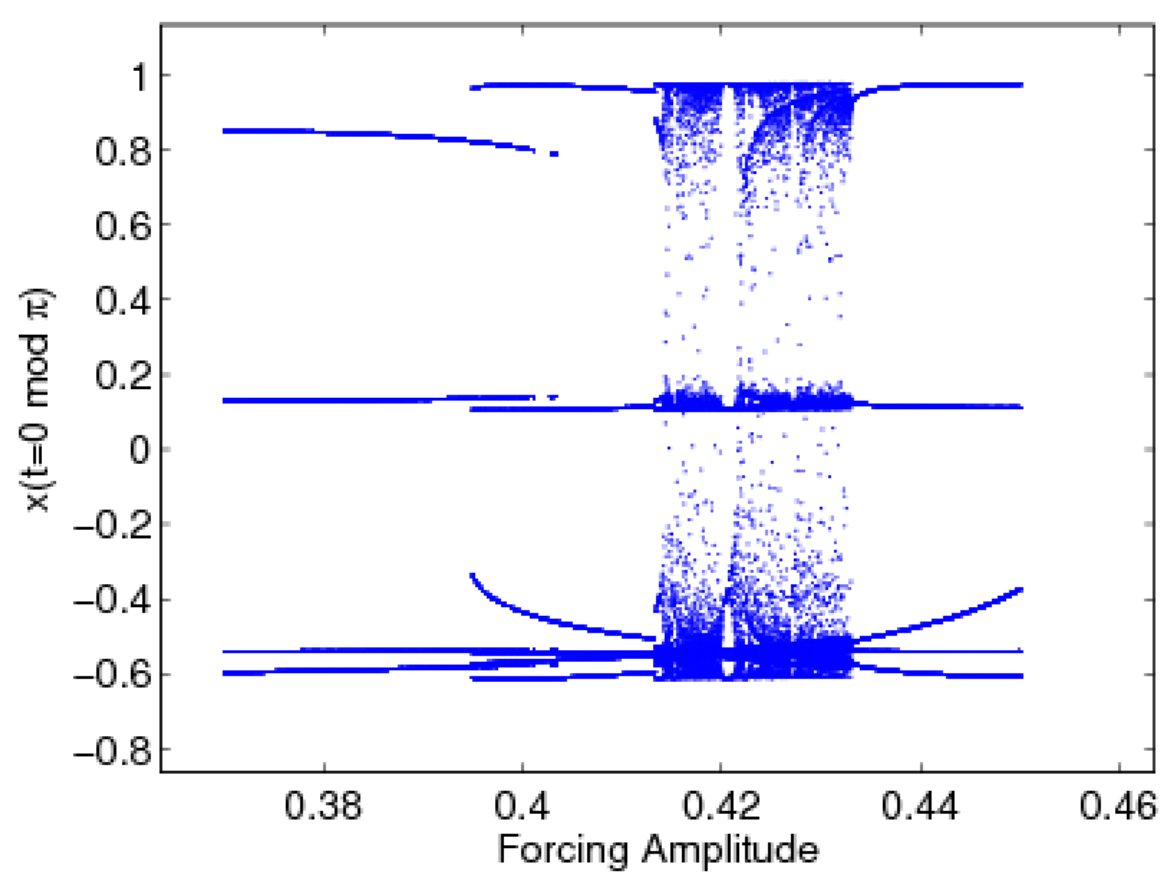

Figure 1: Bifurcation diagram of BVP equations (1)-(2) sampled every $t=0 \bmod \pi$ with $a=0.7, b=0.8, c=3$, and $\omega=2$. To produce this figure, the equations were integrated sufficiently long to eliminate the transient behaviour, the $x$ coordinate was sampled every $\pi$ units of time. 


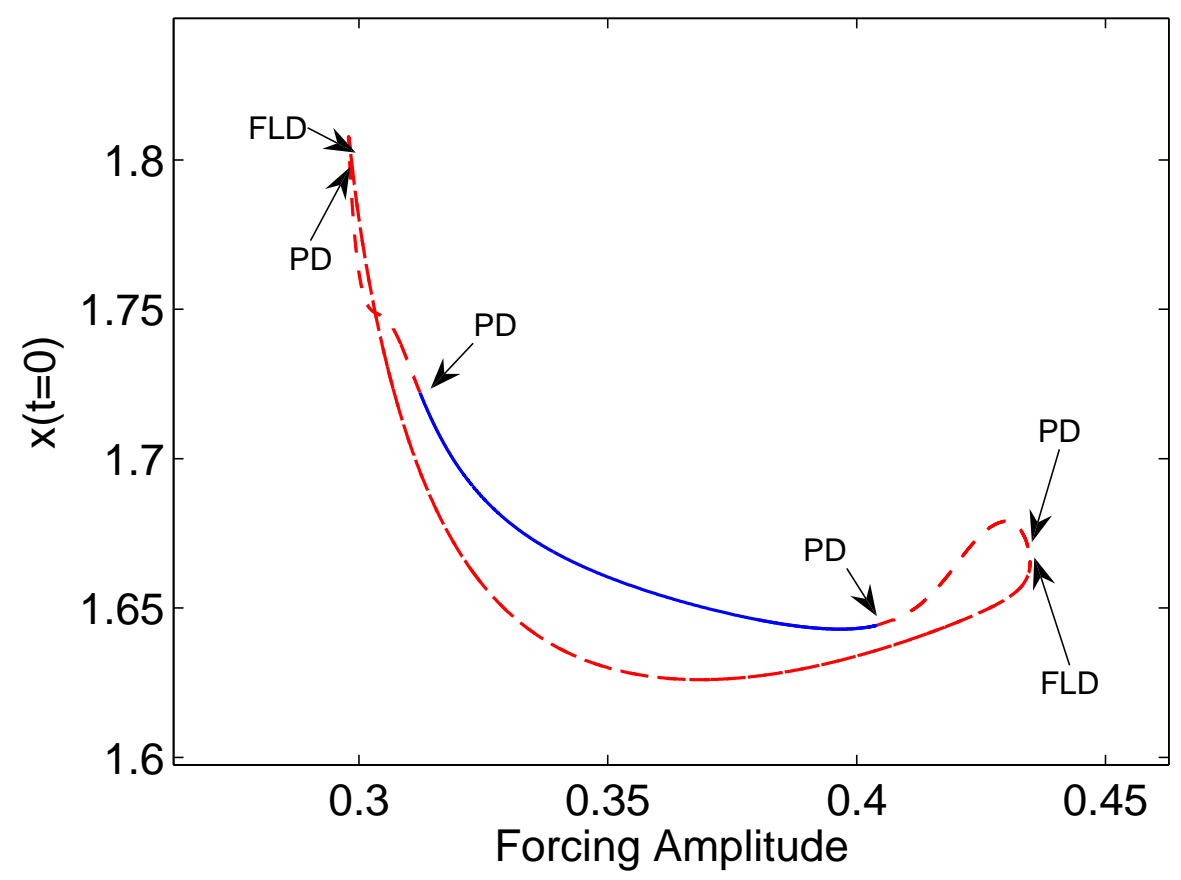

Figure 2: Continuation of $4 \pi$ periodic solution via Galerkin method. Stable periodic solutions are represented as a solid blue line and unstable periodic solutions are represented as a dashed red line. Period doubling bifurcations (PD) and fold bifurcations (FLD) are also shown. The parameters used were the same as those used in Figure 1. 
Recall that overlapping period $4 \pi$ and $6 \pi$ solutions are observed in Figure 1. Due to the nature of the bifurcation diagram's construction it only displays stable solutions, therefore we have an incomplete understanding of the dynamics. To obtain the full dynamics of the solution branch, we also need to track the unstable branch and determine the type of bifurcations that occur. In Figure 2 we have a complete description of the period $4 \pi$ solution and its local bifurcations.

The eigenvalues of the monodromy matrix were determined at each computed point of the solution curve. This identifies any local bifurcations. Floquet theory states that an eigenvalue beyond the unit circle indicates an unstable solution and if all eigenvalues are within the unit circle the solution is termed stable [15]. Moreover, we can identify the type of local bifurcation by the way an eigenvalues leaves the unit circle. If an eigenvalue leaves via -1 it denotes a period doubling bifurcation, if it passes through +1 it represents a fold or symmetry breaking bifurcation and if a pair of eigenvalues leave the unit circle as complex conjugates then a secondary Hopf bifurcation occurs. The period $4 \pi$ branch has period doubling bifurcations at $A=0.435,0.404$, 0.312 and 0.298 . We also observe fold bifurcations at $A=0.435$ and 0.298 ; see Figure 2. We now have a full understanding of the dynamics of the period $4 \pi$ solution with the added advantage of knowing the locations of the different bifurcations. By applying our suite of continuation methods we uncover the dynamics of periodic solutions which remained uncertain from the study of Barnes and Grimshaw [1].

\section{Characterising chaotic behaviour via the 0-1 test}

Gottwald and Melbourne [7] recently introduced a test for chaos known as the 0-1 test. Rather than trying to characterise the rate at which solutions to a dynamical system diverge, as in the computation of Lyapunov exponents, 
they exploit an ergodic property of chaotic attractors. The main advantage of this method is that it circumvents the need for phase space reconstruction. For data derived experimentally we do not, necessarily, have direct access to the dynamical equations, in such cases traditional methods attempt to estimate the Lyapunov exponents via the embedding theory of Takens [16]; see also the work of Gottwald and Melbourne [7] for additional references. However, for the 0-1 test, all that is required is an 'observable' from the dynamics sampled at equal times, almost any reasonable function of the state of the system will do. This makes the 0-1 test an especially easy test to use. The test determines if a variable (to be defined later as $p(n)$ ), derived from the time series data, resembles Brownian motion.

The 0-1 test uses the observable to drive the dynamics on a well chosen group extension, and then exploits a theorem from Nicol et al. [14] that states that the dynamics on the group extension is bounded if the underlying dynamics is non-chaotic, but is unbounded and sub-linear if the dynamics is chaotic. In particular, $p(n)$ exhibits Brownian motion (asymptotically) when the underlying dynamics is chaotic [14].

Using a modified version of the original test, as modified by Gottwald and Melbourne [8], let $\phi(n)$ be an observable on our set of $N$ data points (obtained from integrating DEs, PDEs or from experimental data) and randomly choose a $\varpi \in \mathcal{R}$, any random number in $(0,2 \pi)$ is sufficient. If $p(n)$ is defined by

$$
p(n)=\sum_{j=1}^{n} \phi(j) \cos (j \varpi), \quad n=1,2,3, \ldots, N,
$$

then $p(n)$ will exhibit Brownian motion as a function of $n$ when the dynamics are chaotic. For Brownian motion we expect the average of $|p(j+n)-p(j)|$ to grow like $\sqrt{n}$ for $j=1,2,3, \ldots$ Therefore, the square of $|p(j+n)-p(j)|$ should asymptotically approach linear growth with $n$ for sufficiently large $N$. 
Gottwald and Melbourne [8] define the mean-square displacement

$$
M(n)=\frac{1}{N-n} \sum_{j=1}^{N-n}[p(j+n)-p(j)]^{2}, \quad n=1,2,3, \ldots .
$$

Provided $n \ll N$ we expect $M(n)$ to scale linearly and is characterised via the computation of

$$
K=\lim _{n \rightarrow \infty} \frac{\log M(n)}{\log n} .
$$

$K=0$ indicates a non-chaotic data set while a value of $K=1$ indicates a chaotic data set, hence the name ' $0-1$ ' test. In practice we compute the slope of a linear regression of $\log M(n)$ against $\log n$.

Figures 3a (0-1 test) and 3b (Lyapunov spectrum) show near perfect correlation between the 0-1 test and the Lyapunov exponent test for forcing amplitudes $A>0.13$. However, for $A<0.13$ there is an example of a phenomenon discussed by Gottwald and Melbourne [8] where the frequency of the underlying dynamics (quasi-periodic in this case) is commensurate with the choice of frequency $\varpi$, resulting in extreme values. These extreme values occur in Figure 3a for forcing between 0.1 and 0.13, there is also a significant peak between 0 and 0.05. For forcing amplitude between 0.19 and 0.21 Figure 3a displays extreme values, where the Lyapunov exponent in $3 \mathrm{~b}$ suggests quasi-period motion. Figure 3c confirms that forcing between 0.19 and 0.21 is non-chaotic. In these cases we have $p(n)=a_{0} * n+\cdots$ which is understood from the Fourier decomposition of $\phi(j)$. When $\phi(j)$ is multiplied by $\cos (\varpi j)$ the Fourier decomposition will have a term like $a_{0}$ for all $j$, as $e^{+i j \varpi} \times e^{-i j \varpi}=1$ (personal communication, Gottwald). Therefore $|p(j+n)-p(j)|^{2} \sim\left|a_{0} \times(j+n)-a_{0} \times(j)\right|^{2}=a_{0}^{2} n^{2}$. Consequently, the mean square displacement will have quadratic growth, resulting in a value of $K=2$. However, unless the frequencies are exactly commensurate longer time series will eventually result in a value of $K=0$, although the convergence may be very slow. 


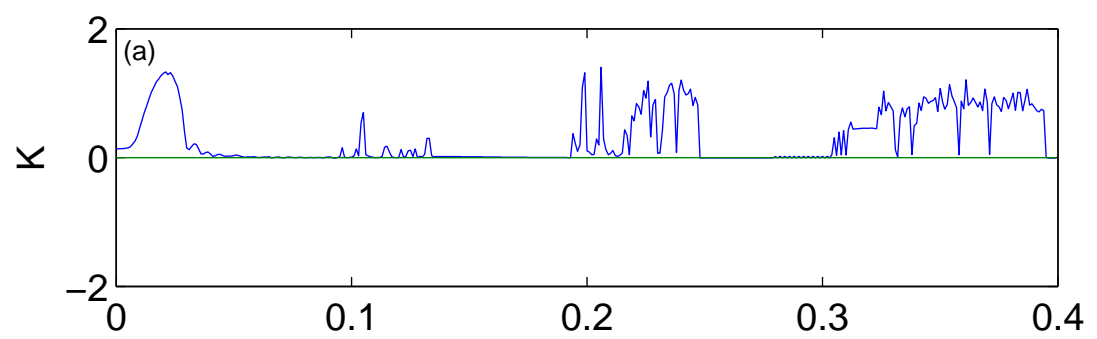

Forcing Amplitude (A)

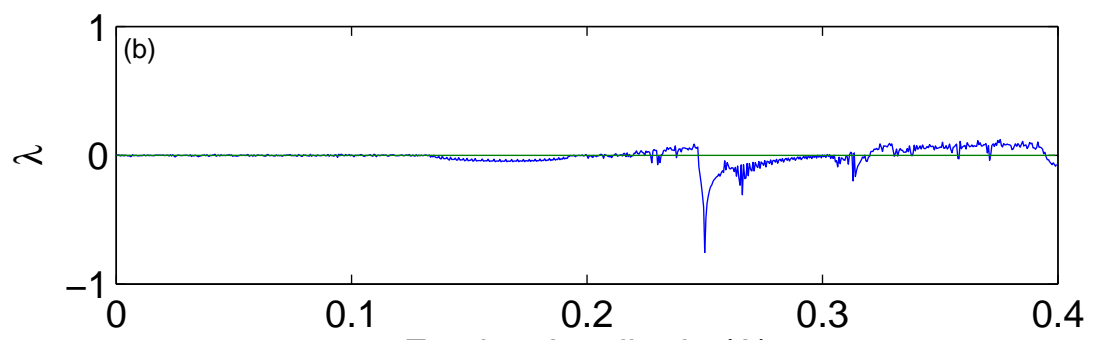

Forcing Amplitude (A)

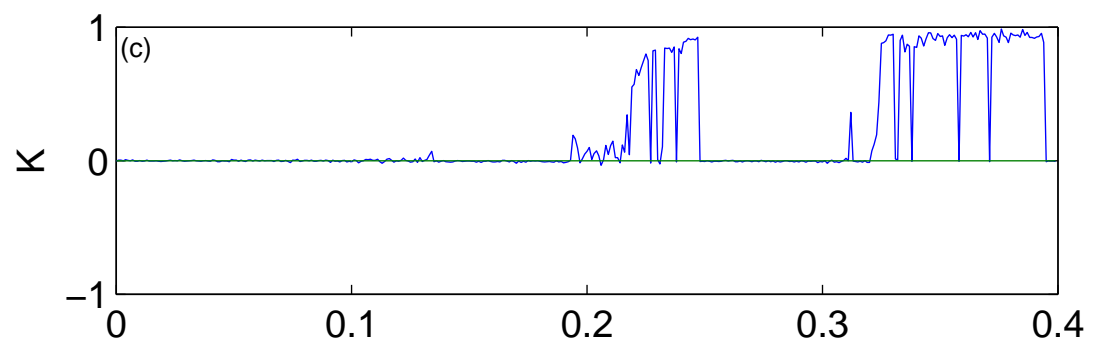

Forcing Amplitude (A)

Figure 3: Comparison of the 0-1 test (a) and (c) with the Lyapunov Spectrum (b). Only one fixed value of $\varpi$ was used in the computation of the 0-1 test in (a), whereas 100 random values of $\varpi$ were used in (c). This highlights the resonances described in the work of Gottwald and Melbourne [8], and hence the need to use multiple $\varpi$. We know from Barnes and Grimshaw [1] that the dynamics between $0<A<0.13$ is quasi-periodic and hence likely to produce resonances. System parameters are $a=0, b=0.8, c=3$, $\omega=1.5$. The observable, $\phi$, used for the above computations was $\phi(j)=x(j)+y(j)$. The time series was 10000 points, sampled every 2.72 units of time, with an initial transient of 15000 units of time, integrated before the time series was recorded. 
The computation of $K$ illustrated in Figure 3a used only a single fixed value of $\varpi$ at each sampled forcing amplitude. To overcome the slow convergence Gottwald and Melbourne [8] took multiple random $\varpi$ and computed $K$ for each $\varpi$ and took the median of the resulting set of $K \mathrm{~s}$. The median is preferred over the mean so that the occasional extreme value does not skew the results.

Figure $3 \mathrm{c}$ illustrates the same numerical experiment as Figure 3a except that now 100 random values of $\varpi$ are taken and the median extracted, for each sampled forcing amplitude (Gottwald and Melbourne [8] suggest 100 randomly chosen $\varpi$ should be sufficient). Figure 3c shows that there is now complete agreement with the Lyapunov exponents in Figure 3b. Taking the multiple values of $\varpi$ eliminates the effects of the resonances on the computation of the 0-1 test, for the case of quasi-periodic motion.

This example illustrates another advantage of the 0-1 test over the Lyapunov test in that for quasi-periodic motion the Lyapunov exponent hovers above and below the horizontal axis leading to uncertainty in the type of dynamics, whether weakly chaotic or non-chaotic. However, the 0-1 test, when multiple $\varpi$ are taken, clearly distinguishes between chaotic and non-chaotic dynamics. If there is any remaining uncertainty as to the status of a parameter point, increasing the number of solution points will drive $K$ to 0 or 1 , thereby removing the uncertainty. Although we are able to do the same for the Lyapunov exponent approach, increasing the time of integration, the end result may still remain ambiguous. Nevertheless, near the forcing amplitude of 0.19 to 0.21 there may be a question as to the status of the dynamics. The slightly elevated value of $K$ in Figure $3 \mathrm{c}$ in this parameter range may cause concern. However, this is resolved by taking larger number of data points. As an illustration, let us take the parameter value at 0.195 , which was slightly elevated, and used a larger number of data points for the computation of $K$. At 10,000 points we had a value of 0.1266 , at 20,000 points a value of 0.066 and at 30,000 points we had a value of 0.022 . It seems clear that the value of $K$ is converging to 0 as the number of points gets large. Therefore the 
dynamics at this point is non-chaotic, suggesting quasi-periodicity.

\section{Conclusion}

By utilising continuation and Floquet methods we have extended the analysis of the the BVP system studied in the work of Barnes and Grimshaw [1], thus providing a better understanding of the system's dynamics. In particular we are able to explain the type of bifurcations that occur at critical points and track unstable solutions. This information is difficult to attain using only bifurcation diagrams. We also verified that the 0-1 test for chaos accurately determines when the BVP system exhibits chaotic dynamics. Even the more challenging case of quasi-periodic motion is well handled using this test with minor modification. We feel that the $0-1$ test offers a number of advantages over the Lyapunov exponent test including its ease of use, and its ability to distinguish clearly between chaotic and non-chaotic dynamics.

Acknowledgement We thank G. A. Gottwald for helpful comments.

\section{References}

[1] B. Barnes and R. Grimshaw, Numerical studies of the periodically forced Bonhoeffer van der Pol system, International Journal of Bifurcation and Chaos, 7(12):2653-2689, 1997. C628, C630, C633, C636, C638

[2] A. Ben-Tal, D. Shein, S. Zissu, Studying ferroresonance in actual power systems by bifurcation diagram,Electric Power Systems Research, 49, 175-183, 1999. doi:10.1016/S0378-7796(98)00117-5. C630 
[3] N. Britton, Essential Mathematical Biology, Springer Undergraduate Mathematics Series, Springer-Verlag, 2003. C628

[4] H. Croisier, P. Dauby, Continuation and bifurcation analysis of a periodically forced excitable system, Journal of Theoretical Biology, 246:430-448, 2007. doi:10.1016/j.jtbi.2007.01.017. C628

[5] E. Doedel and R. Heinemann, Numerical computation of periodic solution branches and oscillatory dynamics of the stirred tank reactor with $\mathrm{A} \rightarrow \mathrm{B} \rightarrow \mathrm{C}$ reactions, Chemical Engineering Science, 38(9):1493-1499, 1983. doi:10.1016/0009-2509(83)80084-0. C629

[6] R. Fitzhugh, Impulses and physiological states in theoretical models of nerve membrane, Biophysical Journal, 1:445-466, 1961. http://www. pubmedcentral.nih.gov/articlerender . fcgi?artid=1366333 C627, C628

[7] G. A. Gottwald, I. Melbourne, A new test for chaos in deterministic systems,Proc. Royal Soc. Lon. Series A, 460:603-611, 2004. doi:10.1098/rspa.2003.1183. C629, C633, C634

[8] G. A. Gottwald, I. Melbourne, Testing for chaos in deterministic systems with noise, Physica D, 212:100-110, 2005. doi:10.1016/j.physd.2005.09.011. C634, C635, C636, C637

[9] J. Guckenheimer, K. Hoffman and W. Weckesser, The forced van der Pol equation 1: The slow flow and its bifurcations, SIAM J. Applied Dynamical Systems, 2(1):1-35, 2003. doi:10.1137/S1111111102404738. C629

[10] H. Hayashi, S. Ishizuka, M. Ohta and K. Hirakawa, Chaotic behavior in the onchidium giant neuron under sinusoidal stimulation, Physics Letters, 88A:435-438, 1982. doi:10.1016/0375-9601(82)90674-0. C628

[11] A. L. Hodgkin, A. F. Huxley, A quantitative description of membrane current and its application to to conduction and excitation in 
nerve,J. Physiol., 117:500-544, 1952. http://www.pubmedcentral . nih.gov/articlerender.fcgi?artid=1392413 C627

[12] D. Li, J. Xu, A method to determine the periodic solution of the nonlinear dynamics system, Journal of Sound and Vibration, 275:1-16, 2004. doi:10.1016/S0022-460X(03)00656-4. C629

[13] A. Nayfeh and B. Balachandran, Applied Nonlinear Dynamics - Analytical, Computational, and Experimental Methods, Wiley Series in Nonlinear Science, John Wiley \& Sons, 1995. C629

[14] M. Nicol, I. Melbourne and P. Ashwin, Euclidean extensions of dynamical systems, Nonlinearity, 14:275-300, 2001. doi:10.1088/0951-7715/14/2/306. C629, C634

[15] R. Seydel, Practical Bifurcation and Stability Analysis From Equilibrium to Chaos, Interdisciplinary Applied Mathematics, Springer-Verlag, 1994. C630, C633

[16] F. Takens, Detecting strange attractors in turbulence, Lecture Notes in Mathematics - Springer, 898:366-381, 1981. C634

[17] M. Urabe and A. Reiter, Numerical computation of nonlinear forced oscillations by Galerkin's procedure, Journal of Mathematical Analysis and Applications, 14:107-140, 1966. C629 


\section{Author addresses}

1. R. M. Pennifold, School of Physical, Environmental and Mathematical Sciences, University of New South Wales at the Defence Force Academy, Australia. mailto:r.pennifold@adfa.edu.au

2. H. S. Sidhu, School of Physical, Environmental and Mathematical Sciences, University of New South Wales at the Defence Force Academy, Australia.

3. G. N. Mercer, School of Physical, Environmental and Mathematical Sciences, University of New South Wales at the Defence Force Academy, Australia. 